\title{
Paroxysmal Hypertension Induced by an Insulinoma
}

\author{
Ko Harada ${ }^{1}$, Yoshihisa Hanayama ${ }^{1}$, Kou Hasegawa ${ }^{1}$, Masaya Iwamuro ${ }^{1}$, Hideharu Hagiya ${ }^{2}$, \\ Ryuichi Yoshida $^{3}$ and Fumio Otsuka ${ }^{1}$
}

\begin{abstract}
Insulinoma is a rare, usually benign, pancreatic neuroendocrine tumor. The clinical features of an insulinoma are fasting hypoglycemia with neuroglycopenic symptoms including confusion and unusual behavior, while hypertension is usually not associated with the disease. We herein report a patient with insulinoma who manifested paroxysmal hypertension and neuroglycopenic symptoms. The possible etiology of hypertension induced by an insulinoma is catecholamine release in response to hypoglycemia, which may cause acute hypertension through activation of the sympatho-adrenal system. This case implies that sustained hyperinsulinemia due to insulinoma can be functionally linked to the induction of paroxysmal hypertension.
\end{abstract}

Key words: insulinoma, hypertension, hypoglycemia

(Intern Med 56: 413-417, 2017)

(DOI: 10.2169/internalmedicine.56.7758)

\section{Introduction}

Insulinoma is a rare pancreatic islet cell tumor with an estimated incidence of 0.4 per 100,000 person-years (1). The characteristic clinical manifestation of insulinoma is fasting hypoglycemia with neuroglycopenic symptoms including confusion, visual changes, and unusual behaviors (2). However, hypeinsulinemia has not been generally considered to be a causal factor in the development of hypertension (3-5). We herein report a rare case of hypoglycemia-induced paroxysmal hypertension that was ameliorated by the surgical removal of an underlying insulinoma.

\section{Case Report}

A 65-year-old woman was admitted to our hospital because of episodes of altered mental status and high blood pressure. Initially, the patient had been referred due to uncontrolled blood pressure rising to $196 / 114 \mathrm{mmHg}$ during her dental treatments. Forty days prior to admission, the patient was taken to the emergency department of another hospital with an altered mental status and hypertension despite the fact that etizolam had been administered for stress- induced hypertension. At that time, her blood pressure was 226/129 mmHg, while her blood glucose level was $48 \mathrm{mg}$ / dL. A solution of $50 \%$ dextrose in water was administered intravenously, resulting in improvement in the patient's level of consciousness. After that, the patient suffered four similar attacks with high systolic blood pressure $>200 \mathrm{mmHg}$ as well as lowered plasma glucose $(\mathrm{PG})<70 \mathrm{mg} / \mathrm{dL}$. Since her blood pressure at home was $154 / 92 \mathrm{mmHg}$ on average, treatment with $25 \mathrm{mg}$ losartan was commenced. Four days prior to admission, she had been transported to another hospital due to an altered mental status, with a very low glucose level of $25 \mathrm{mg} / \mathrm{dL}$ and inappropriately high level of immunoreactive insulin (IRI) of $19.8 \mu \mathrm{l} / \mathrm{mL}$. She was then referred to our hospital.

Her blood pressure on admission was 140/94 $\mathrm{mmHg}$, pulse rate was 84 beats/min, respiratory rate was 20/min, and axillary body temperature was $36.6{ }^{\circ} \mathrm{C}$. The patient was alert and oriented to person, place, and time. Her lungs were clear to auscultation bilaterally, and her heart sounds were regular, without any cardiac murmur. Her abdomen was soft and flat without tenderness. A neurological examination revealed no abnormalities. Laboratory tests on admission showed the following values: hemoglobin A1c, 4.5\%; fasting plasma glucose (FPG), $55 \mathrm{mg} / \mathrm{dL}$; IRI, $45.2 \mu \mathrm{U} / \mathrm{mL}$; and $\mathrm{C}$

\footnotetext{
${ }^{1}$ Department of General Medicine, Okayama University Graduate School of Medicine, Dentistry and Pharmaceutical Sciences, Japan, ${ }^{2}$ Division of Infection Control and Prevention, Osaka University Hospital, Japan and ${ }^{3}$ Department of Gastroenterological Surgery, Okayama University Graduate School of Medicine, Dentistry and Pharmaceutical Sciences, Japan Received for publication May 24, 2016; Accepted for publication June 19, 2016 Correspondence to Dr. Ko Harada, me422084@s.okayama-u.ac.jp
} 

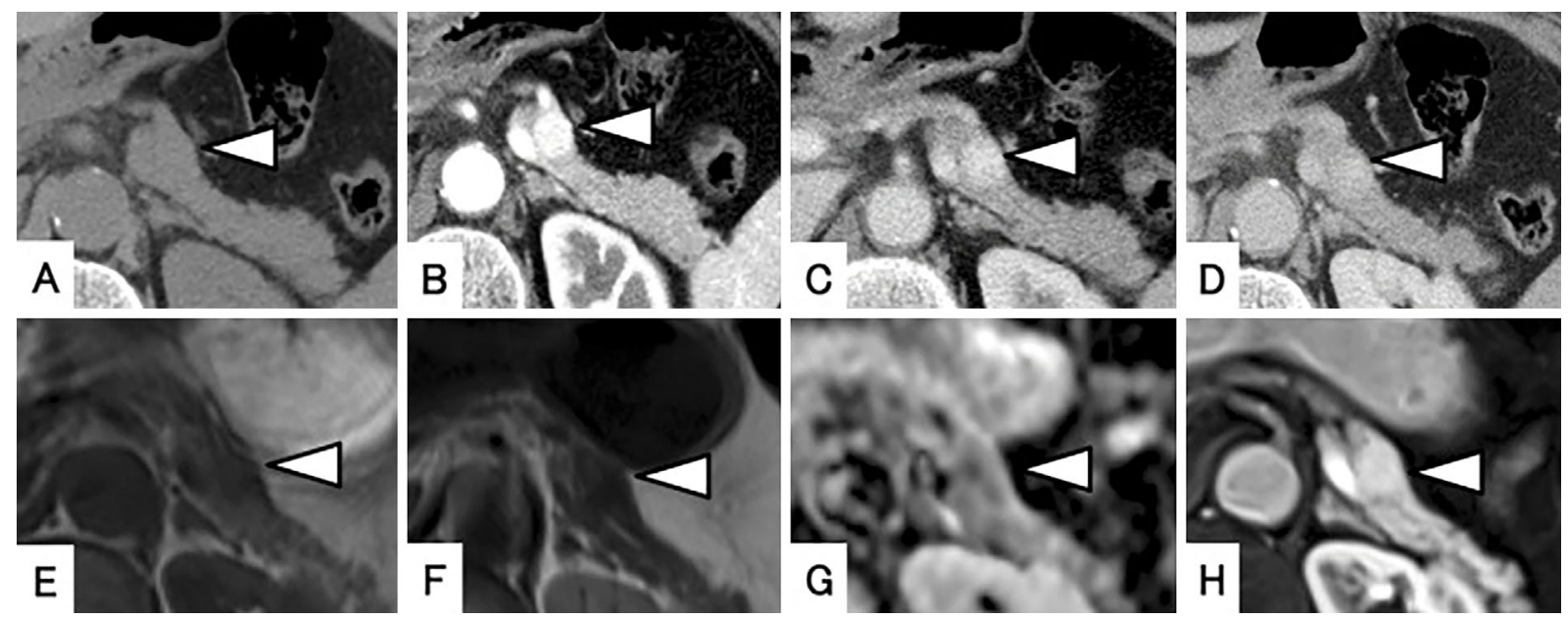

Figure 1. The radiological findings of a pancreatic tumor. A contrast-enhanced CT image of the abdomen demonstrating a lesion $15 \mathrm{~mm}$ in diameter in the pancreatic body (A: arrowhead). The tumor was strongly enhanced in the arterial phase (B: arrowhead) and slightly enhanced in the portal phase (C: arrowhead) and venous phase (D: arrowhead). T1-weighted (E) and T2-weighted (F) MRI and diffusion-weighted imaging (G) showed a lesion in the pancreatic body that had a low signal (arrowheads). The tumor was slightly enhanced in a gadolinium-enhanced image (H: arrowhead).

peptide, $4.4 \mathrm{ng} / \mathrm{mL}$. By endocrine workup, no dysfunction of the anterior pituitary, thyroid, or adrenal glands was found. The diagnostic indicators of insulinoma, including the Fajan: IRI/PG $>0.3$, Grunt: PG/IRI $<2.5$, and Taminato indices: $(100-P G) \times($ IRI-3) $\geq 280$, were positive, at 0.792 , 1.26, and 1,260, respectively, while the Turner index IRIX 100/(PG-30)>200 was negative, at 180.8. In addition, the patient had Whipple's triadic signs: hypoglycemic symptoms, low glucose level at the time of the symptoms, and the amelioration of the symptoms after glucose administration. After admission, glucose $30 \mathrm{mg} / \mathrm{h}$ was administered intravenously via a peripherally inserted central catheter, considering her several hypoglycemic episodes. Octreotide administration reduced the serum IRI and C peptide levels by $33 \%$ and $39 \%$, respectively, for 2 hours. Enhanced abdominal computed tomography (CT) was performed and showed a lesion $15 \mathrm{~mm}$ in diameter in the pancreatic body that was strongly enhanced in the arterial phase (Fig. 1A-D). Magnetic resonance imaging (MRI) of the abdomen showed a lesion in the pancreatic body, exhibiting a low signal on T1- and T2images and slight enhancement on the Gd-enhanced image (Fig. 1E-H). Significant accumulation of the tracer was not detected in the pancreatic tumor by ${ }^{18} \mathrm{~F}$-fluorodeoxyglucose positron emission tomography/CT.

Based on the diagnosis of a solitary insulinoma, laparoscopic resection of the pancreatic body and tail was performed. There was no evidence of gross invasion, abnormal lymph nodes, or metastases. The resected specimen contained a well-circumscribed white round mass, $15 \mathrm{~mm}$ in diameter, in the pancreatic body with the absence of a surrounding capsule (Fig. 2A). The mass was pathologically found to be a well-differentiated tumor with amyloid deposition showing $1 \% \mathrm{Ki}-67$-positive cells and $<2$ mitoses (/10 high-power field (HPFs)) (Fig. 2B). Immunohistochemical studies showed positive staining for insulin and the neuroendocrine markers synaptophysin and chromogranin A but not for glucagon or gastrin (Fig. 2C-E), leading to the diagnosis of the insulinoma as a grade 1 neuroendocrine tumor based on the 2010 WHO Classification for Pancreatic Endocrine Neoplasms (6).

After surgery, the patient's FPG and IRI normalized to $116 \mathrm{mg} / \mathrm{dL}$ and $5.3 \mu \mathrm{l} / \mathrm{mL}$, respectively. Her systolic blood pressure (SBP) and diastolic blood pressure (DBP) during hospitalization gradually normalized (Fig. 3). The established indicators for insulinoma, including the Fajan, Grunt, and Turner indexes, were normal, at 0.046, 21.9, and 33.1, respectively. The levels of plasma noradrenaline (NA) and urinary NA also normalized after surgery. She has not had paroxysmal hypertension that seemed to be related to hypoglycemia since the surgery. Although administration of antihypertensive agents was discontinued 1 week after the surgery, her SBP has been maintained at less than $140 \mathrm{mmHg}$, and her home blood pressure (100-110 mmHg) and mental condition have also clearly stabilized.

\section{Discussion}

We report a case of insulinoma manifesting paroxysmal hypertension. Insulinoma is a rare, usually benign, pancreatic neuroendocrine tumor that can occur sporadically or as a part of the MEN type $1(2,7)$. The majority of patients with an insulinoma are between 30 and 60 years of age, with women accounting for $59 \%$ of the patients $(8,9)$. The most common clinical features of an insulinoma are fasting hypoglycemia with discrete episodes of neuroglycopenic symptoms (3). Interestingly, our patient showed both hypoglycemic episodes and paroxysmal hypertension.

Hyperinsulinemia accompanying insulin resistance has 

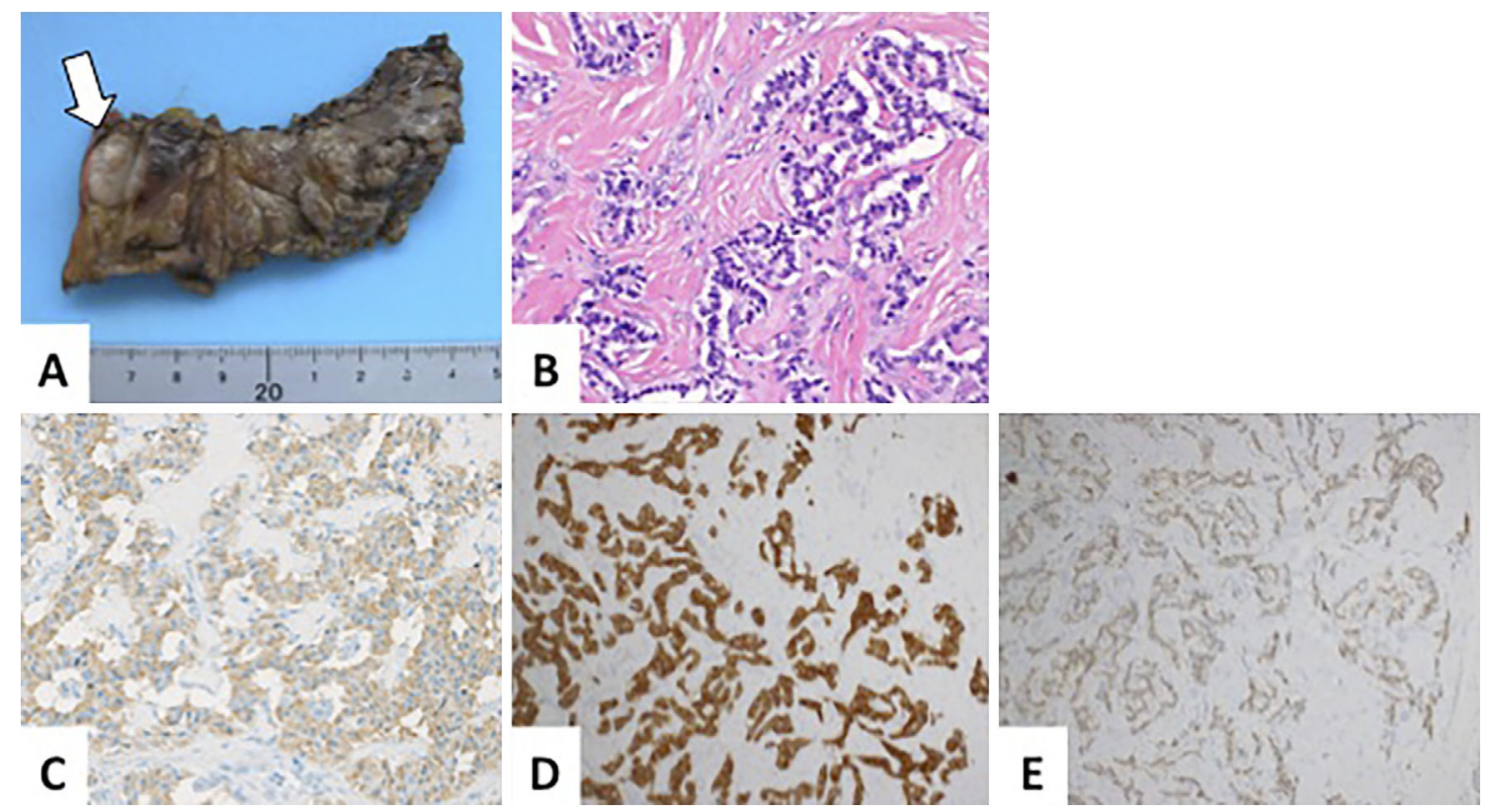

Figure 2. The pathological findings of the resected insulinoma. The pancreatectomy specimen contained a well-circumscribed white round mass $15 \mathrm{~mm}$ in diameter in the pancreatic body with the absence of a surrounding capsule (A: arrow). In Hematoxylin and Eosin staining, a well-differentiated tumor with amyloid deposition was observed histopathologically (B). The tumor had $1 \%$ Ki67-positive cells per 10 HPFs and less than 2 mitoses per 10 HPFs. Immunostaining was positive for insulin (C). Immunostaining for synaptophysin (D) and chromogranin A (E) was also positive. The tumor was thus classified as a grade 1 neuroendocrine tumor based on the WHO classification.

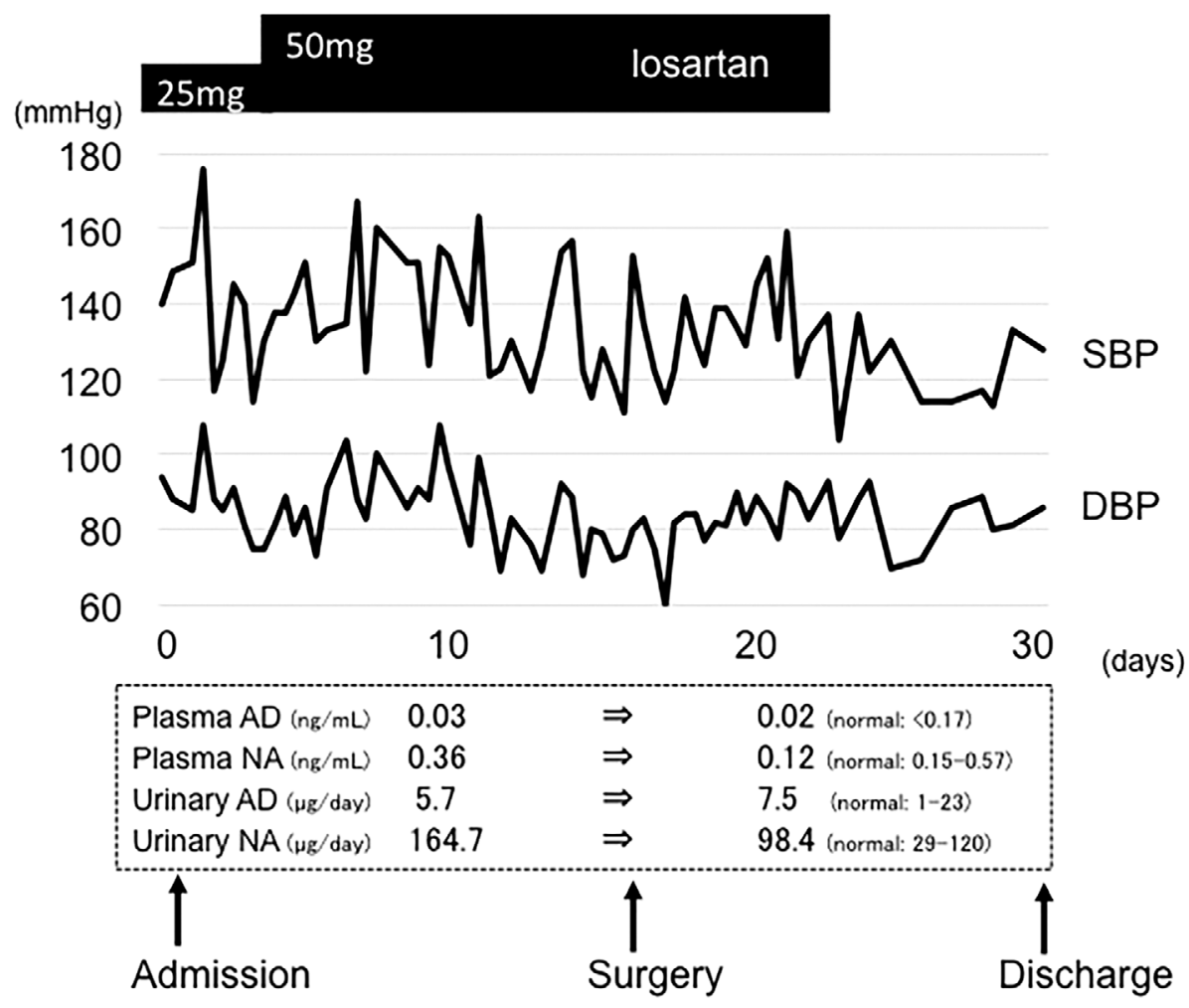

Figure 3. Clinical course. The systolic blood pressure (SBP) and diastolic blood pressure (DBP) during hospitalization and the plasma and urinary adrenaline (AD) levels and noradrenaline (NA) level are shown. The levels of plasma NA and urinary NA were decreased after surgery. Although administration of losartan was discontinued 1 week after surgery, the SBP remained lower than 140 mmHg. 
been reported to be associated with hypertension $(10,11)$. However, most of the studies conducted in the 1990s showed that there is no association between hyperinsulinemia and hypertension in the setting of insulinoma $(4,5,12-14)$. To cite an example, Sawicki et al. investigated the hypothesis that insulin is a causal and independent risk factor for blood pressure elevation in humans by comparing pre- and post-operative blood pressures of 34 patients with insulinoma. They concluded that correction of hyperinsulinemia after surgery for insulinoma did not result in blood pressure changes (5). To our knowledge, there is only one documented report regarding insulinoma accompanying significant hypertension. Kaul et al. reported that a 10-year-old girl presented with hypoglycemia with a high insulin level, distal symmetrical motor-sensory axonal neuropathy and hypertension, although her urine catecholamine levels were normal. That patient's hypertension showed remarkable improvement one year after tumor excision (15).

There are a few possible mechanisms of hypertension caused by insulinoma. First, hypoglycemia due to insulinoma can induce catecholamine release, which may cause paroxysmal hypertension through activation of the sympathoadrenal system (16). Tsujimoto et al. reported that onethird of 414 patients with diabetes who presented with hypoglycemia to the emergency room had severe hypertension higher than $180 / 120 \mathrm{mmHg}$. Their article suggested that catecholamine hypersecretion as a result of severe hypoglycemia might contribute to severe hypertension (17). Our patient also showed high plasma and urinary levels of catecholamine on admission. In addition, the reduction in the plasma and urinary sodium after surgery indicated that the removal of the insulinoma impaired the secretion of catecholamine.

Another hypothesis is that the sodium-retaining effect of insulin may contribute to a rise in blood pressure (18). Accumulating data have shown that insulin stimulates renal sodium reabsorption primarily at the distal nephron, playing an important role in the development of hypertension and possibly of salt sensitivity (19). In our case, this hypothesis is, in part, supported by the fact that the patient's home BP was stabilized after tumor excision without any medication. Our case exhibited both symptoms of neurologic features due to hypoglycemia and emergent hypertension with SBP $>180 \mathrm{mmHg}$. These symptoms might have been challenging for the emergent unit to differentiate from cerebrovascular disorders such as cerebral hemorrhage or infarction (20).

Collectively, we experienced a case of insulinoma with paroxysmal hypertension as well as neuroglycopenic symptoms, despite the fact that hypertension is not usually considered to be related to insulinoma. A hypoglycemic state should therefore always be considered when a patient manifests acute hypertension and neurologic symptoms, such as an altered mental status.

The authors state that they have no Conflict of Interest (COI).

\section{References}

1. Service FJ, McMahon MM, O’Brien PC, Ballard DJ. Functioning insulinoma--incidence, recurrence, and long-term survival of patients: a 60-year study. Mayo Clin Proc 66: 711-719, 1991.

2. Service FJ, Dale AJ, Elveback LR, Jiang NS. Insulinoma: clinical and diagnostic features of 60 consecutive cases. Mayo Clin Proc 51: 417-429, 1976.

3. O’Brien T, Young WF, Palumbo PJ, O'Brien PC, Service FJ. Hypertension and dyslipidemia in patients with insulinoma. Mayo Clin Proc 68: 141-146, 1993.

4. Pontiroli AE, Alberetto M, Pozza G. Patients with insulinoma show insulin resistance in the absence of arterial hypertension. Diabetologia 35: 294-295, 1992.

5. Sawicki PT, Heinemann L, Starke A, Berger M. Hyperinsulinaemia is not linked with blood pressure elevation in patients with insulinoma. Diabetologia 35: 649-652, 1992.

6. Klimstra DS, Modlin IR, Coppola D, Lloyd RV, Suster S. The pathologic classification of neuroendocrine tumors: a review of nomenclature, grading, and staging systems. Pancreas 39: 707-712, 2010.

7. Placzkowski KA, Vella A, Thompson GB, et al. Secular trends in the presentation and management of functioning insulinoma at the Mayo Clinic, 1987-2007. J Clin Endocrinol Metab 94: 1069-1073, 2009.

8. Halfdanarson TR, Rubin J, Farnell MB, Grant CS, Petersen GM. Pancreatic endocrine neoplasms: epidemiology and prognosis of pancreatic endocrine tumors. Endocr Relat Cancer 15: 409-427, 2008.

9. Grant CS. Surgical aspects of hyperinsulinemic hypoglycemia. Endocrinol Metab Clin North Am 28: 533-554, 1999.

10. Feskens EJ, Tuomilehto J, Stengård JH, Pekkanen J, Nissinen A, Kromhout D. Hypertension and overweight associated with hyperinsulinaemia and glucose tolerance: a longitudinal study of the Finnish and Dutch cohorts of the Seven Countries Study. Diabetologia 38: 839-847, 1995.

11. Haffner SM, Miettinen H, Gaskill SP, Stern MP. Metabolic precursors of hypertension. The San Antonio Heart Study. Arch Intern Med 156: 1994-2001, 1996.

12. Leonetti F, Iozzo P, Giaccari A, et al. Absence of clinically overt atherosclerotic vascular disease and adverse changes in cardiovascular risk factors in 70 patients with insulinoma. J Endocrinol Invest 16: 875-880, 1993.

13. Fujita N, Baba T, Tomiyama T, Kodama T, Kako N. Hyperinsulinaemia and blood pressure in patients with insulinoma. BMJ 304: $1157,1992$.

14. Tsutsu N, Nunoi K, Kodama T, Nomiyama R, Iwase M, Fujishima M. Lack of association between blood pressure and insulin in patients with insulinoma. J Hypertens 8: 479-482, 1990.

15. Kaul B, Kaur P, Tripathi M, et al. An unusual cause of reversible axonal neuropathy and hypertension in a 10-year-old girl. J Clin Neurosci 19: 1196-1197, 2012.

16. Hepburn DA, Deary IJ, Frier BM, Patrick AW, Quinn JD, Fisher BM. Symptoms of acute insulin-induced hypoglycemia in humans with and without IDDM. Factor-analysis approach. Diabetes Care 14: 949-957, 1991.

17. Tsujimoto T, Yamamoto-Honda R, Kajio H, et al. Vital signs, QT prolongation, and newly diagnosed cardiovascular disease during severe hypoglycemia in type 1 and type 2 diabetic patients. Diabetes Care 37: 217-225, 2014.

18. Brands MW, Manhiani MM. Sodium-retaining effect of insulin in diabetes. Am J Physiol Regul Integr Comp Physiol 303: R1101R1109, 2012.

19. Sarafidis PA, Bakris GL. The antinatriuretic effect of insulin: an unappreciated mechanism for hypertension associated with insulin resistance? Am J Nephrol 27: 44-54, 2007. 
Intern Med 56: 413-417, 2017 DOI: 10.2169/internalmedicine.56.7758

20. Johnson W, Nguyen ML, Patel R. Hypertension crisis in the emergency department. Cardiol Clin 30: 533-543, 2012.
The Internal Medicine is an Open Access article distributed under the Creative Commons Attribution-NonCommercial-NoDerivatives 4.0 International License. To view the details of this license, please visit (https://creativecommons.org/licenses/ by-nc-nd/4.0/).

(C) 2017 The Japanese Society of Internal Medicine http://www.naika.or.jp/imonline/index.html 